\title{
Optical Sky Monitoring: Past and Future
}

\author{
H.-J. Bräuer ${ }^{1}, N$. Vogt $^{2,3}$ \\ 1 Sternwarte Sonneberg, 96515 Sonneberg, Germany \\ 2 Max-Planck-Institut für Astrophysik, 85740 Garching, Germany \\ 3 Universidad Catolica de Chile, Santiago, Chile
}

\begin{abstract}
After outlining the achievements of photographic sky monitoring at Sonneberg Observatory we describe a concept of a future CCD-based sky patrol. We suggest a world-wide network of 6 identical observing stations which cover the entire sky down to a limiting magnitude of $\sim 18^{m}$ and with a time resolution of between 8 and 24 hours. A preliminary technical design is proposed, and the astrophysical and general impact of such a project is briefly discussed.
\end{abstract}

\section{Introduction}

To be on alert for every incident in the sky is a task of mankind, an essential in any civilisation on some cultural level. Even the people of ancient times knew that: there are records left by antiquity on novae, supernovae and comets, among them the Chinese and Korean reports, which are of scientific value up to the present day.

For millenia visual observation was the only means of monitoring. Photographic techniques in astronomy became common practice just about one hundred years ago. Only since then was it possible to run what we understand by a systematic sky patrol. It was conducted first at Harvard Observatory (USA) and, a few decades later, by Cuno Hoffmeister in Sonneberg, which now is the site with the largest uninterrupted records.

\section{The Sonneberg archive}

The Sonneberg patrol was started in 1926 as one of the cornerstones of the Sonneberg programme of variable star research. It consists of a continuous record of the whole northern sky (Sky Patrol in the literal sense) and, in addition, of 81 fields selected along or near the Milky Way (Field Patrol). One quarter of all variables known in the Galaxy were detected by its means. Today its archive contains about 250,000 photographic plates. It is an excellent source of information on the long-term behaviour of active objects, and a valuable support of observation made from satellites at non-optical wavelengths. Its logbook data are computerised and retrievable from a database. 
Table 1. Sonneberg Observatory: Photographic telescopes currently in operation. (cf. Bräuer and Fuhrmann, 1992)

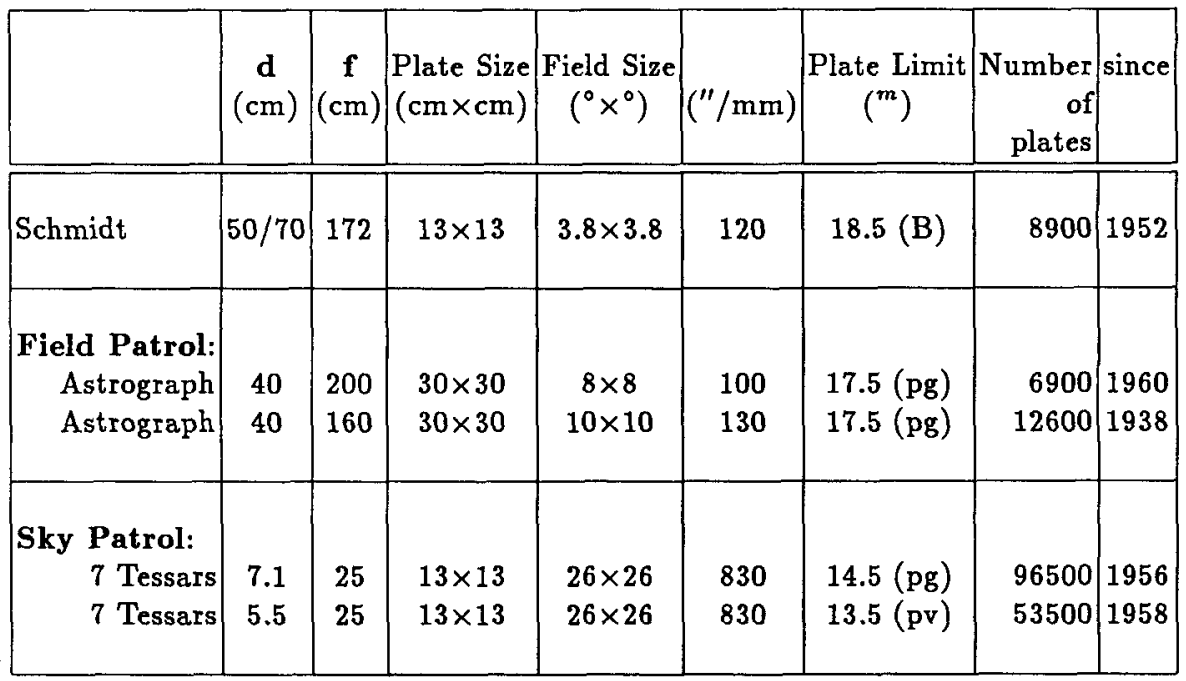

For some time the Sonnebergians have been contemplating a new method of sky patrol, but have not been able to proceed because the observatory has been threatened with closure. Doubtless Sonneberg, with the involvement of the nearby Technische Universität Ilmenau, would be the place where this new patrol could materialize the fastest and in the most natural way. The new patrol can however be realized anywhere else, albeit with far more difficulty. The following is an outline of the project.

\section{ASPA: The new way}

An efficient sky patrol requires utilization of the most advanced technology available. These days the place of the photographic plate is being taken by digital detectors. CCDs have begun to dominate nearly all areas of observational astronomy, and now they are on the verge of conquering even the last stronghold of the traditional photographic plate: wide-field observation. Thus, since the time has come to develop new concepts of a CCD-based sky patrol, an international working group, called ASPA (All Sky Patrol Astrophysics) was recently founded by one of us (N.V.) together with Constanze la Dous (Madrid) and Hanns Ruder (Tübingen). The main aims of ASPA are:

1. to monitor the flux variations (stellar and non-stellar objects) in the entire sky with a time resolution of $8-24$ hours, using latest-generation digital detectors (CCDs);

2. to continue the traditional photographic sky patrol until CCD observation is in operation; 
3. to give rapid and effective on-line access to the variations observed at any sky position to the astronomical community; and

4. to transform existing photographic plate archives into digital form in order to offer the same access to data taken in past decades.

ASPA should be conducted by a world-wide network of 6 identical semiautomatic observing stations. Each station would consist of about 24 cameras ( $D \approx 0.2 \mathrm{~m}, f \approx 0.6 \mathrm{~m}$ ), possibly on two mountings: one for regions near the equator, the other for those near the pole. If each camera is equipped with a $2048 \times 2048$ pixel CCD, a limiting magnitude of $18^{m}$ with a photographic accuracy of 3 - $5 \%$ could be reached with exposure times of the order of 6 minutes (field size $3^{\circ} \times 3^{\circ}$, scale $5^{\prime \prime} /$ pixel, average distance between stars: 10 pixels). The above estimates are based on Kroll and Lehmann (1993). The observing stations should be located at established observatories, preferably some $120^{\circ}$ apart from each other in longitude. Possible locations on the northern hemisphere could be the Hawaiian Islands (Mauna Kea?), the Canary Islands and a far-east location. On the southern hemisphere they could be in Chile (Paranal area: cooperation with the Catholic University, Santiago), Namibia (Gamsberg?) and Australia. Those stations are only for observing. All data should be, possibly in compressed form, transmitted to an ASPA Centre for reduction, analysis, storage and distribution. The centre should, if possible, be an active observatory where the observing stations can be tested before deployment, and maintenance tasks as well as further technical developments can be done. The centre should also be responsible for the digitization of photographic plate archives.

\section{A preliminary technical design}

There are many ways of realizing a CCD-based sky patrol technically. We will only present some first ideas. (In our description we consider the northern hemisphere case.)

Each observing station could consist of two units:

Unit A: 16 cameras on a single parallactic mounting, movable only about its polar axis. With each camera adjusted to one fixed declination, they cover the declination zones $-6^{\circ} \ldots+39^{\circ}$.

Unit B: 8 additional cameras, also on a single parallactic mounting, but each one adjustable to two different declination zones (three zones for the pole camera). Each camera is equipped with a step motor for fine adjustment in right ascension. Those cameras cover the declination zones $+42^{\circ} \ldots+90^{\circ}$.

By this arrangement, each accessible sky position could be observed once per night with the following procedure: if we assume an average length of an observing night of 10 hours, and a range in right ascension covered from $\mathrm{HA}=$ $+4^{h}$ (west) at beginning of night to $\mathrm{HA}=-4^{h}$ (east) at its end, a total range of $18^{h}$ in right ascension would be monitored in a total exposure time of 600 minutes. Unit A would perform this task for the equatorial zones, resulting in 90 fields of $3^{\circ} \times 3^{\circ}$ per night and camera, with 6 minutes exposure each. Nearer 
Table 2. Proposed distribution of cameras in Unit B

\begin{tabular}{|l|c|rr|rr||c|}
\hline & Camera & First Zone & Second Zone & Total \\
& No & $\delta$ & $N_{e}$ & $\delta$ & $N_{e}$ & $N_{e}$ \\
\hline \multirow{3}{*}{ Distribution of cameras } & 1 & $+90,+87^{\circ}$ & 9 & $+42^{\circ}$ & 69 & 78 \\
$\left(N_{e}=\right.$ number of exposures & 2 & $+84^{\circ}$ & 12 & $+45^{\circ}$ & 66 & 78 \\
necessary to cover & 3 & $+81^{\circ}$ & 17 & $+48^{\circ}$ & 63 & 80 \\
$\Delta \alpha=18^{\text {h }}$ in one night $)$ & 5 & $+78^{\circ}$ & 21 & $+51^{\circ}$ & 59 & 80 \\
& 6 & $+75^{\circ}$ & 26 & $+54^{\circ}$ & 55 & 81 \\
& 7 & $+72^{\circ}$ & 30 & $+57^{\circ}$ & 51 & 81 \\
& 8 & $+69^{\circ}$ & 35 & $+60^{\circ}$ & 47 & 82 \\
& $+66^{\circ}$ & 39 & $+63^{\circ}$ & 43 & 82 \\
\hline
\end{tabular}

to the pole, where unit $B$ is active, each camera would be able to cover two different declination zones. Table 2 gives a possible assignment of those zones to the cameras:

The exposures in all cameras of unit B should be synchronized. Between exposures, its mounting is pointed at the same hour angle as unit $A$, while the actual declination zone and the accurate field centre in right ascension are selected for each camera. During exposure the mounting just follows the daily motion of the stars.

Mechanics and optics of this design would be relatively simple, especially in unit A. This unit could work either in pointed or in tracking mode. We would obtain about 2100 frames $\left(3^{\circ} \times 3^{\circ}\right)$ per night and station, corresponding to about 18 GByte of information. The network of 6 stations would imply a time resolution of about 8 hours (perfect weather conditions assumed) and an overlap between northern and southern stations of $15^{\circ}$ in declination along the equator.

\section{Astrophysical impact, outlook}

It is rather evident that observations of this kind open fascinating new aspects covering wide areas in present-day astrophysics. This refers not only to the 'classical' variable star work on pulsating, eruptive, or eclipsing variables. Targets of ASPA will range from objects on the fringe of the visible universe like quasars and AGNs down to tiny earth-orbit crossing asteroids nearer than the Moon. These are a few highlights: Many thousands of galaxies will be searched for supernovae and we may expect about five detections per night. One of the mysteries of modern astrophysics is the nature of gamma-ray bursts and their hitherto undetected optical counterparts. If, for instance, they would be brighter than $14^{\mathrm{m}}$ for a few second at least, ASPA might record one coincidence of gamma-ray burst and its optical counterpart every 100 days. We also would probably observe between 50 and several hundreds of gravitational lensing events per year. Near-earth asteroids of diameters as small as $60 \mathrm{~m}$ would be detectable, and so on and so forth.

Of course, this project implies rather ambitious goals. Challenging features are the development and optimization of the organization of observations, espe- 
cially in unit $B$, which requires quite sophisticated software in order to ensure efficient automatic operation. The second main challenge is the reduction and management of the large amount of data: in total some $\mathbf{2 4 0}$ million stars are to be automatically monitored, reduced, classified and, if variable, placed at the astronomical communities disposal within 24 hours. It is an immense task, but it is not impossible with today's technology: presently active gravitaional microlensing experiments (EROS, MACHO, OGLE) toward the Magellanic Clouds and the bulge of the Milky Way also produce large amounts of information on stellar and non-stellar variability. They have already developed part of the technology, and it should be utilized by ASPA. For instance, the MACHO group is able to perform the photometric reduction of a night's data (5 GByte) within 24 hours (Cook et al., 1994). This corresponds already to about $30 \%$ of the data expected from an ASPA observing station. Sophisticated reduction procedures which work automatically and assure adequate photometric accuracies are also available (Schechter et al., 1993).

In general terms, ASPA will obtain astrophysically relevant and important information at relatively low cost. It is unique information, and, if ASPA is not carried out, this information could never be recovered later, however sophisticated future technologies will be. The service function of ASPA to the astronomical community implies that a large range of scientists and institutions will benefit from the project. In particular, a very close interaction between groundbased and space astronomy is to be expected. Finally, the technological aspects of the project require close cooperation between astronomers and technology researchers at universities, as well as with industry. Interdisciplinary cooperation will be very important.

\section{References}

Bräuer H.-J., Fuhrmann B., 1992, The Messenger 68, 24

Cook K. et al., 1994, IAU XXIInd General Assembly, Poster Abstracts, p. 138

Kroll P., Lehmann T., 1993, IAU WG on Wide-Field Imaging, Newsletter 3, 30

Schechter P.L., Mateo M., Saha A., 1993, PASP 105, 1342

M. Merck: 1) You will get some 60 TByte a year of data. How do you plan to store them? 2) CR events are difficult to suppress if you don't have 2 contemporaneous observations of the same field. Are you planing to implement this type of observations?

N. Vogt: 1) On optical disks or other similar, may be future, storage devices.

2) This would even double the data received. In the tracking mode these events are unimportant. In pointing mode we still have to find a solution. 